\title{
Imaging signatures of the local density of states in an electronic cavity
}

\author{
Carolin Gold $\odot,{ }^{1, *}$ Beat A. Bräm $\odot,{ }^{1}$ Michael S. Ferguson $\odot,{ }^{2}$ Tobias Krähenmann $\odot,{ }^{1}$ Andrea Hofmann, ${ }^{1}$ Richard Steinacher, ${ }^{1}$ \\ Keith R. Fratus, ${ }^{3,4}$ Christian Reichl, ${ }^{1}$ Werner Wegscheider, ${ }^{1}$ Dietmar Weinmann ${ }^{\circ},{ }^{3}$ Klaus Ensslin (1), ${ }^{1,5}$ and Thomas Ihn ${ }^{1,5}$ \\ ${ }^{1}$ Solid State Laboratory, ETH Zurich, 8093 Zurich, Switzerland \\ ${ }^{2}$ Institute for Theoretical Physics, ETH Zurich, 8093 Zurich, Switzerland \\ ${ }^{3}$ Université de Strasbourg, CNRS, Institut de Physique et Chimie des Matériaux de Strasbourg, UMR 7504, F-67000 Strasbourg, France \\ ${ }^{4}$ HQS Quantum Simulations, Haid-und-Neu-Str. 7, 76131 Karlsruhe, Germany \\ ${ }^{5}$ Quantum Center, ETH Zurich, $\mathrm{CH}-8093$ Zurich, Switzerland
}

(Received 27 November 2020; accepted 9 April 2021; published 6 July 2021)

\begin{abstract}
We use scanning gate microscopy to study electron transport through an open, gate-defined resonator in a $\mathrm{Ga}(\mathrm{Al}) \mathrm{As}$ heterostructure. Raster-scanning the voltage-biased metallic tip above the resonator, we observe distinct conductance modulations as a function of the tip position and voltage. Quantum-mechanical simulations reproduce these conductance modulations and reveal their relation to the partial local density of states in the resonator. Our measurements illustrate the current frontier between possibilities and limitations in imaging the local density of states in buried electron systems using scanning gate microscopy.
\end{abstract}

DOI: 10.1103/PhysRevResearch.3.L032005

\section{INTRODUCTION}

Scanning gate microscopy (SGM) provides a unique mean to investigate local properties of carrier transport in semiconductor nanostructures based on buried two-dimensional electron gases (2DEG) [1,2]. This imaging technique uses the capacitive coupling between the voltage-biased metallic tip scanned above the sample surface and the electrons in the 2DEG. Successfully imaged local phenomena and systems in various materials range from disorder-induced or engineered localized states [3-12], magnetic focusing of electrons [13,14], quantum rings [15-18], quantum Hall edge states [19], ballistic as well as viscous regimes of interacting electron liquids [20], to the milestone observation of branched electron flow [21-23]. One major goal of SGM is the experimental measurement of the local density of states in nanostructures. Here, SGM provides a unique opportunity, as only few scanning probe experiments allow one to image the local density of states directly, and the most successful technique, scanning tunneling microscopy, requires the $2 \mathrm{DEG}$ to be accessible at the surface.

The imaging of the local density of states with SGM is well studied in theory $[15,24,25]$ and approached experimentally by a number of pioneering experiments [16,21-23,2628]. However, a major obstacle for the experimental realization of scanning gate measurements of truly local electron properties is the invasiveness of the tip-induced potential, which alters the quantum states of interest. Imaging local

\footnotetext{
*cgold@phys.ethz.ch

Published by the American Physical Society under the terms of the Creative Commons Attribution 4.0 International license. Further distribution of this work must maintain attribution to the author(s) and the published article's title, journal citation, and DOI.
}

quantum-mechanical properties, such as the local density of states, with SGM thus requires weakly invasive tip potentials. Unfortunately, the measurement signals obtained in the latter are too weak to be resolved unless strongly confined systems are investigated. These, in turn, lead to a significant loss of spatial resolution of the SGM measurement [29].

Recently, an accommodation for these two competing requirements of weakly invasive tip potential and sufficient signal strength was found [26]. An open resonator structure of intermediate size [30-32] confines only fundamental onedimensional cavity modes which can be uniquely identified and addressed [33]. This holds true even in slightly less open resonators [26]. In the latter, the moderate confinement and size of the cavity allows for SGM imaging with tip potentials smaller than the Fermi energy and sufficient spatial resolution [26].

In this Letter, we demonstrate the correlation between the partial local density of states in such an open resonator structure with the conductance modulations observed in SGM measurements. We scan the voltage-biased metallic SGM tip above the structure for both weakly and strongly invasive tip voltages, and observe distinct conductance modulations in the area of the resonator. Quantum-mechanical simulations not only exhibit a good qualitative agreement with the experimental data but also display a correlation between the conductance modulations in the SGM conductance maps and the partial local density of states in the cavity. These results show that weakly invasive SGM provides a tool for measuring direct signatures of the partial local density of states in large two-dimensional electronic structures.

\section{SAMPLE AND EXPERIMENTAL SETUP}

Our measurements are performed at $T=270 \mathrm{mK}$ using the measurement setup depicted schematically in Fig. 1(a). The SGM tip is raster-scanned above a $\mathrm{Ga}(\mathrm{Al}) \mathrm{As}$ 
(a)

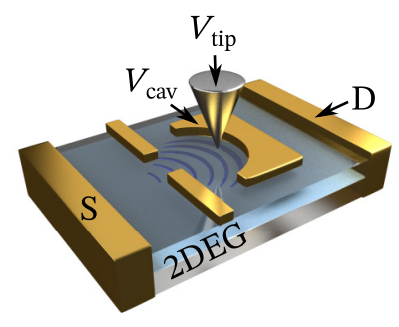

(b)
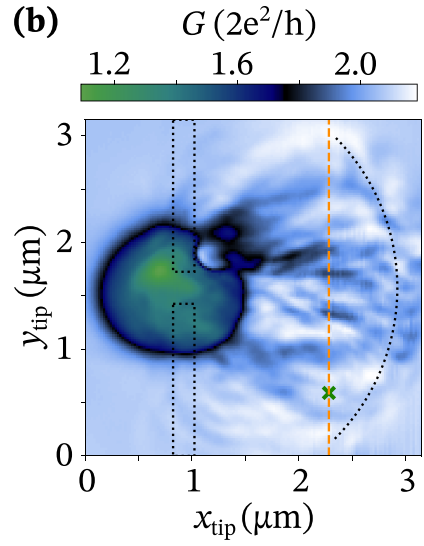

FIG. 1. (a) Schematic of the measurement setup. Voltage-biased metallic gates (golden) on a $\mathrm{Ga}(\mathrm{Al}) \mathrm{As}$ heterostructure form an open resonator structure (schematic blue standing wave pattern) in the two-dimensional electron gas (blue). A SGM tip is positioned above the structure to measure the conductance between source $(S)$ and drain (D) ohmic contacts as a function of the tip position. (b) SGM image $G(x, y)$ of the cavity area for tip voltage $V_{\text {tip }}=-1 \mathrm{~V}$ and cavity gate voltage $V_{\text {cav }}=-400 \mathrm{mV}$. Dotted lines outline the approximate position of the Schottky gates. Measurements in Fig. 2 are performed along the orange dashed line [Fig. 2(b)]; respectively, the green crossed point [Fig. 2(a)].

heterostructure, in which a 2 DEG resides $90 \mathrm{~nm}$ underneath the surface. Applying suitable voltages to the lithographically defined metallic Schottky gates allows us to form an open resonator for electrons by depleting the 2 DEG underneath the gates.

The back and forth reflection of electrons between the quantum point contact (QPC) through which they are injected into the cavity and the arc-shaped cavity gate enhances the local density of states in the resonator as compared to an open 2DEG in the absence of the cavity. The length $(2 \mu \mathrm{m})$ and opening angle $\left(90^{\circ}\right.$, defined by the arc of the cavity gate) of the resonator render its area significantly larger than any characteristic lateral size of the SGM tip-induced potential. This avoids the insufficient spatial resolution observed for SGM inside smaller closed structures [29].

Throughout the measurements, the QPC is set to the third conductance plateau (in absence of the cavity) and the resonator is formed by applying a cavity-gate voltage which depletes the 2DEG underneath the gate. In this configuration, the resonator supports more than 50 populated, radial, spindegenerate modes [34].

We perform SGM measurements over the whole cavity area defined by the QPC gates on one side and the cavity gate on the other side. By raster-scanning the voltage biased tip at a height of $h_{\text {tip }} \approx 80 \mathrm{~nm}$ above the sample surface, we measure the differential conductance $G(x, y)=d I_{\mathrm{SD}}(x, y) / d V_{\mathrm{SD}}$ as a function of the tip position $(x, y)$ in a two-terminal setup. Here, $I_{\mathrm{SD}}\left(V_{\mathrm{SD}}\right)$ is the current (voltage) between the source and drain ohmic contacts. The resulting conductance $G(x, y)$ for a weakly invasive tip (for which the amplitude of the tip-induced potential is much smaller than the Fermi energy) is depicted in Fig. 1(b). It exhibits conductance modulations in the cavity area, which are in good agreement with previous

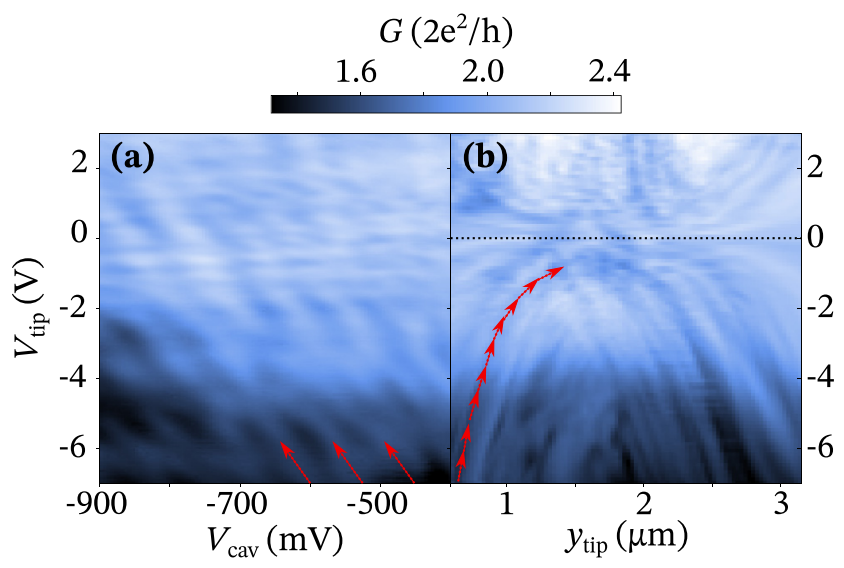

FIG. 2. (a) Differential conductance $G\left(V_{\text {cav }}, V_{\text {tip }}\right)$ as a function of cavity voltage $V_{\text {cav }}$ and tip voltage $V_{\text {tip }}$ at the tip position marked by the green cross in Fig. 1(b). (b) Differential conductance $G\left(y_{\text {tip }}, V_{\text {tip }}\right)$ along the orange dashed line in Fig. 1(b).

measurements on the same sample [26]. Cross-capacitance between the QPC and cavity gates, as well as scattering of electrons by the cavity gate back through the QPC reduce the overall conductance well below $G=3 \times 2 e^{2} / h$.

\section{TIP INFLUENCE ON THE MODULATED CAVITY CONDUCTANCE}

To study the influence of the tip-induced potential on the cavity, we measure the conductance $G\left(V_{\text {cav }}, V_{\text {tip }}\right)$ of the device for various fixed tip positions along the orange dashed line in Fig. 1(b). By tuning the voltage $V_{\text {cav }}$ in a range for which the 2DEG underneath the gate is depleted, we vary the electronic length of the cavity. In contrast, the tip-induced electrostatic potential with maximum value $U_{\mathrm{t}}$ is varied over the full range from strongly invasive tip potentials $\left(U_{\mathrm{t}}>E_{\mathrm{F}}\right.$, where $E_{\mathrm{F}}$ is the Fermi energy) to weakly invasive tip potentials $\left(U_{\mathrm{t}}<\right.$ $\left.E_{\mathrm{F}}\right)$ [26]. Figure 2(a) exemplarily depicts the conductance measured at the tip position marked by the green cross in Fig. 1(b) $\left(y_{\text {tip }}=0.59 \mu \mathrm{m}\right)$. We observe distinct conductance modulations, which are equidistantly spaced and diagonal as a function of the tip $\left(V_{\text {tip }}\right)$ and cavity $\left(V_{\text {cav }}\right)$ voltages, as indicated by the red arrows.

The Fourier transform of the data in Fig. 2(a) reveals a $\lambda_{F} / 2$ periodicity of the conductance modulations [34]. This observation is evidence for quasi-one-dimensional radial cavity modes [33], which are separated in energy [35] by more than $e V_{\mathrm{SD}}$ [34]. Electronic transport through the cavity is modulated by the cavity modes, which are shifted in energy by either the tip-voltage $V_{\text {tip }}$ or the cavity-gate voltage $V_{\text {cav }}$. This leads to the diagonal conductance modulations seen in Fig. 2(a).

In order to improve our understanding of the relationship between the regular conductance modulations in Fig. 2(a) and the seemingly random modulations in Fig. 1(b), we measure the conductance as a function of the tip potential and tip position along the orange dashed line in Fig. 1(b). We plot the resulting conductance $G\left(y_{\text {tip }}, V_{\text {tip }}\right)$ in Fig. 2(b). At strongly invasive, negative tip potentials the overall conductance is reduced similar to the observation in Fig. 2(a). Transitioning from strongly to weakly invasive tip potentials, peaks 
and troughs in the conductance shift towards the middle of the cavity $\left(y_{\text {tip }}=1.6 \mu \mathrm{m}\right)$, thus forming arcs. Ultimately, the conductance modulations disappear for a small range of tip voltages centered around $V_{\text {tip }}=0 \mathrm{~V}$ [cf. dashed line in Fig. 2(b)]. Increasing the tip voltage beyond this region, we observe a similar but mirrored behavior for conductance modulations at positive tip potentials.

At the common axis in Fig. $2\left(V_{\text {cav }}=-400 \mathrm{mV}\right.$, $\left.y_{\text {tip }}=0.59 \mu \mathrm{m}\right)$, the conductance modulations in $G\left(V_{\text {cav }}, V_{\text {tip }}\right)$ line up with the conductance modulations in $G\left(y_{\text {tip }}, V_{\text {tip }}\right)$ (see also Supplemental Material [34]). We conclude that the arcshaped modulations in Fig. 2(b) are related to the discrete radial cavity modes.

\section{UNDERSTANDING THE MODULATED CAVITY CONDUCTANCE}

There are two common approaches to evaluate the transport properties of noninteracting mesoscopic devices, which have been used extensively to understand SGM measurements: semiclassical expansions [22,36-38] and tight-binding calculations [15,39-41]. The former is formulated in terms of the classical trajectories of electrons exiting the QPC. These are then guided onto branches with an increased electron flow due to the random disorder potential generated by the ionized donors [21-23]. The action of a cavity gate leads to a back-folding of the branches, which are simultaneously deflected by the tip-induced potential. Some of us [42] have used a semiclassical approach to theoretically investigate the effect of varying the tip-branch distance and tip voltage, which leads to arc-shaped conductance features in agreement with the experimental observation in Fig. 2(b). Here, we choose to use tight-binding calculations instead, which reproduce both the arc-shaped as well as the $\lambda_{\mathrm{F}} / 2$-periodic conductance modulations in Fig. 2 and provide an intuitive framework to understand the physical processes in terms of cavity modes.

Specifically, we perform our tight-binding calculations using the KWANT package [43]. The gate geometry in the simulations is similar to the experiment and the potential of the gates is modeled according to the potential suggested in Ref. [44]. The magnitude of the potential applied to the QPC gates for all simulations is chosen such that the QPC is set to the third conductance plateau in absence of the cavity. The disorder in the cavity is modeled by remote impurities positioned at a distance $s=60 \mathrm{~nm}$ from the two-dimensional electron gas. Realizing a specific disorder potential configuration [34], we account for the finite thickness of the electron gas as well as its stand-off distance from the $\mathrm{Ga}(\mathrm{Al}) \mathrm{As}$ surface by using the Fang-Howard variational wave function. Furthermore, we include Thomas-Fermi screening of the disorder potential and assume a spatial correlation of the ionized donors to match the high mobility in our sample [34]. The thus modeled electrostatic potential $U$ (in units of the effective Rydberg energy $E_{\mathrm{Ry}}=5.763 \mathrm{meV}$ ) of the system in the absence of the tip is depicted in Fig. 3(a). The tip is included in the simulation via its electrostatic potential induced in the two-dimensional electron gas [26]. Here, we use a (long-ranged) Lorentzian potential motivated by a breadth of previous works $[1,15,41,45]$ though a Gaussian potential leads to similar results [34].
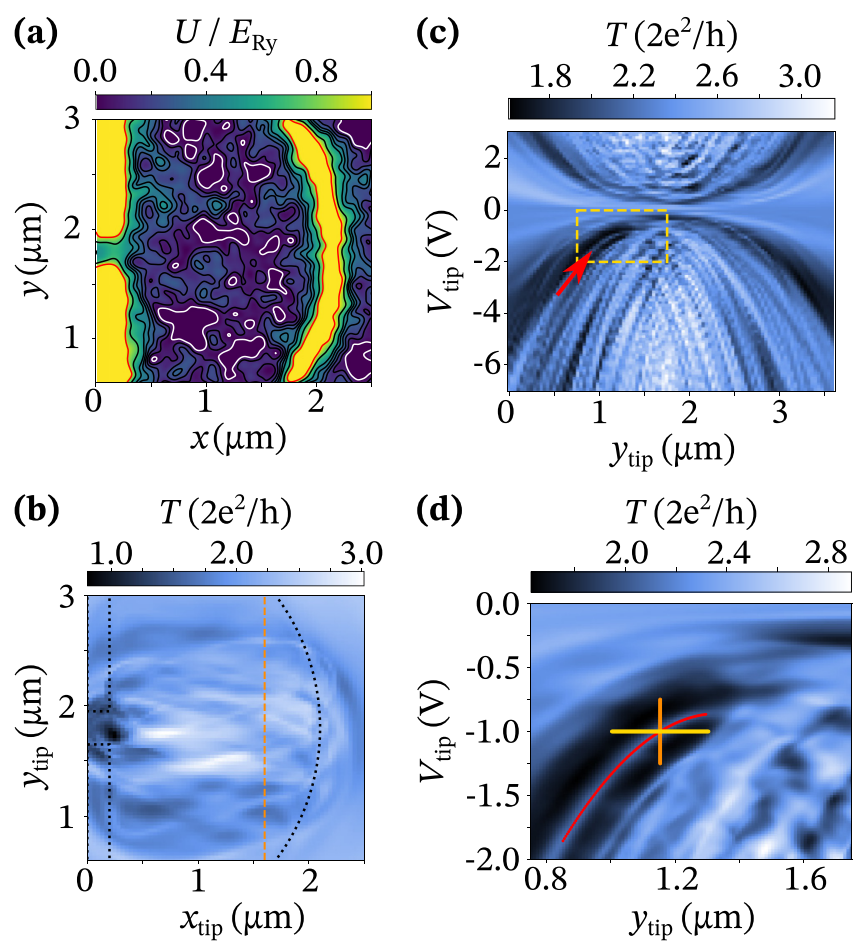

FIG. 3. KWANT simulations of the cavity. (a) Electrostatic potential $U$ (in units of the Rydberg energy $E_{\mathrm{Ry}}$ ) of the cavity in absence of the tip. (b) Numerical SGM data of the transmission through the cavity as a function of the tip position $\left(x_{\text {tip }}, y_{\text {tip }}\right)$ within the cavity area. (c) Numerical transmission as a function of tip voltage and tip position along the dashed orange line in Fig. 3(b). (d) Highresolution calculation of the transmission within the yellow rectangle in Fig. 3(c). The fitted maxima of the parabolic feature of interest are denoted by the red parabola. The simulations depicted in Fig. 4 are performed for the tip parameters denoted by the red arc and the orange/yellow lines.

We perform numerical SGM measurements by calculating the transmission between the source and drain contacts at the Fermi energy as a function of the tip position $\left(x_{\text {tip }}, y_{\text {tip }}\right)$ and the tip voltage $V_{\text {tip. }}$. The calculated equivalents to Figs. 1(b) and 2(b) are shown in Figs. 3(b) and 3(c), respectively. The numerical and physical experiments show striking similarities, with seemingly random modulations in the two-dimensional scan and more regular arc-shaped modulations in the line cut.

We numerically investigate the correlation between these transmission modulations and the cavity modes. To this end, we concentrate on a single conductance maximum, which is isolated from its neighbors and flattens at $y_{\text {tip }} \approx 1.4 \mu \mathrm{m}$ [see Fig. 3(d)]. We calculate the partial local density of states $\mathcal{D}_{\mathrm{P}}$ originating from the source contact in the cavity [46] for each tip parameter along the conductance maximum and along two cuts of fixed tip potential and position, respectively. The tip parameters are chosen such that all three lines cross in a single point $\left(x_{\text {tip }}^{\mathrm{c}}, y_{\text {tip }}^{\mathrm{c}}, V_{\text {tip }}^{\mathrm{c}}\right)$ [see Fig. 3(d)]. The resulting partial local density of states $\mathcal{D}_{\mathrm{P}}\left(x_{\text {tip }}, y_{\text {tip }}, V_{\text {tip }}\right)$ is a two-dimensional map in $(x, y)$ which varies as a function of the parameters $x_{\text {tip }}, y_{\text {tip }}, V_{\text {tip }}[47]$.

To facilitate the comparison of the three different cuts [red, yellow, and orange lines in Fig. 3(d)], we calculate the average 
(a)

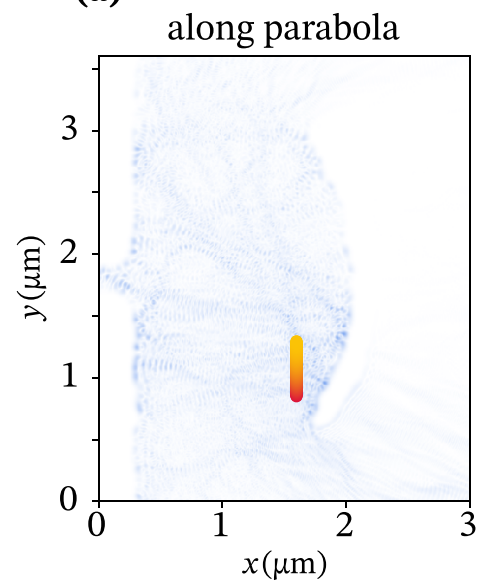

(b)

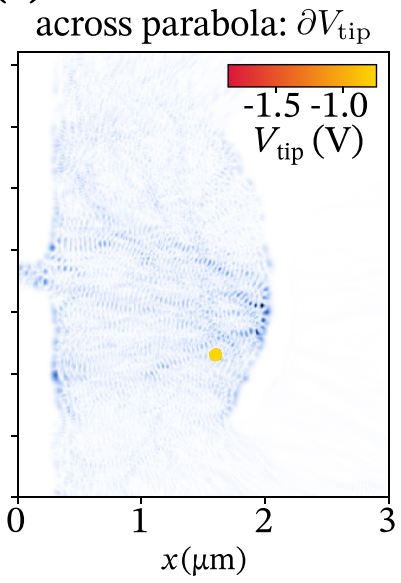

(c)

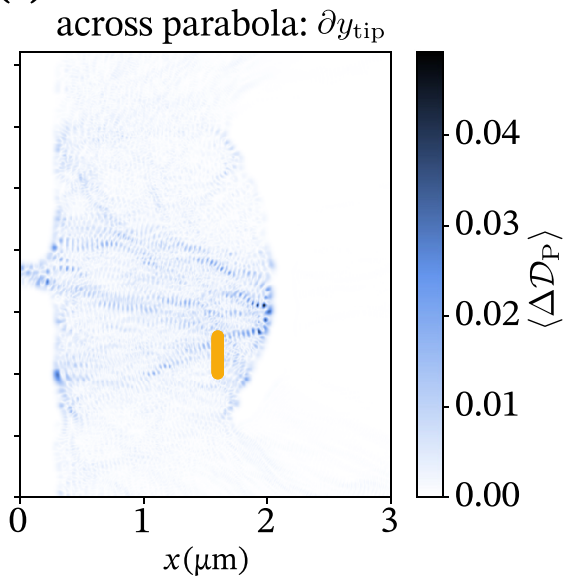

FIG. 4. Spatially resolved variation of the partial local density of states (a) along the conductance maximum, (b) across the conductance maximum for fixed tip position and varying tip voltage, and (c) across the conductance maximum for fixed tip voltage and varying tip position $y_{\text {tip. }}$ The orange-red dots inside the cavity area denote the tip positions and tip voltages for the respective data set.

(over the length of each cut) deviation in the partial local density of states

$$
\begin{aligned}
\left\langle\Delta \mathcal{D}_{\mathrm{P}}\right\rangle & =\left\langle\left|\mathcal{D}_{\mathrm{P}}\left(x_{\text {tip }}^{(i)}, y_{\text {tip }}^{(i)}, V_{\text {tip }}^{(i)}\right)-\mathcal{D}_{\mathrm{P}}\left(x_{\text {tip }}^{\mathrm{c}}, y_{\text {tip }}^{\mathrm{c}}, V_{\text {tip }}^{\mathrm{c}}\right)\right|\right\rangle \\
& =\frac{1}{N} \sum_{i}\left|\mathcal{D}_{\mathrm{P}}\left(x_{\text {tip }}^{(i)}, y_{\text {tip }}^{(i)}, V_{\text {tip }}^{(i)}\right)-\mathcal{D}_{\mathrm{P}}\left(x_{\text {tip }}^{\mathrm{c}}, y_{\text {tip }}^{\mathrm{c}}, V_{\text {tip }}^{\mathrm{c}}\right)\right|,
\end{aligned}
$$

where the index $i$ indicates the points along each of the cuts. We thus obtain in Fig. 4 three two-dimensional plots of the typical spatially resolved variations of $\mathcal{D}_{\mathrm{P}}$ along each of the three curves in parameter space.

Along the conductance maximum [Fig. 4(a)], $\mathcal{D}_{\mathrm{P}}$ changes weakly and uniformly across the cavity. On the other hand, both lines which cross the maximum [Figs. 4(b) and 4(c)] display strong local changes close to $y_{\text {tip }} \approx 1.4 \mu \mathrm{m}$, which indicate a change in the structure of the partial local density of states (cf. Supplemental Material [34]). In terms of cavity modes, we find that the vertical and horizontal cuts through the conductance maximum result in a change of the mode itself. Particularly, we observe that the mode is localized in a region centered around the position of the flattening of the conductance maximum in the simulated SGM measurement in Fig. 3(d). In contrast to this we probe a single mode by following the conductance maximum.

We conclude that the conductance maxima in our experiment are a function of the tip-induced potential and position and contain information about the unperturbed partial local density of states. This demonstrates that weakly invasive SGM measurements in sample geometries like this open resonator do preserve properties of the partial local density of states even in the presence of the tip-induced potential. Furthermore, our data shows that information about the partial local density of states can be resolved also for Lorentzianshaped tip-induced potentials with a full width half maximum of $250 \mathrm{~nm}$.

Both the experimental and numerical data show that most modes feature an enhanced local density of states in the central region of the cavity. Thus, the tip voltage required to tune a specific mode in this region to the Fermi energy is minimal and the transmission features are almost flat along the arc.

In contrast to this, a small variation in the tip positions in regions close to the edges of the cavity requires a larger tip voltage difference to tune a certain cavity mode to the Fermi energy. We thus understand the origin of the arc-shaped modulations observed both in the experimental [Fig. 2(b)] as well as the theoretical data [Fig. 3(c)].

\section{CONCLUSION}

The scanning gate measurements in the open resonator structure presented in this Letter reveal distinct conductance modulations as a function of the cavity-gate voltage $V_{\text {cav }}$, the tip position $\left(x_{\text {tip }}, y_{\text {tip }}\right)$, and the tip voltage $V_{\text {tip }}$. Numerical simulations using the KWANT package [43] substantiate the premise that these conductance modulations are related to the cavity modes. While we cannot measure the local density of states directly, the measurements presented in this Letter offer a potential platform to extract information about large-scale $(>250 \mathrm{~nm})$ modulations of the density of states from scanning gate measurements. A possible measurement scheme to achieve the latter would be to repeat the measurement presented in Fig. 2(b) for all tip positions $x_{\text {tip }}$ throughout the cavity. Information about the local density of states and the localization of the modes is then contained in the exact trend of the thus measured curves and can potentially be extracted (for details, see Supplemental Material [34]). This method explores new avenues to gain insights into the partial local density of states in buried electron systems. At the same time, it also illustrates the current frontier between possibilities and limitations in determining the latter via SGM measurements.

\section{ACKNOWLEDGMENTS}

We thank Peter Märki, Thomas Bähler, as well as the staff of the ETH cleanroom facility FIRST for their techni- 
cal support. We also acknowledge financial support by the ETH Zurich Grant No. ETH-38 17-2, the Swiss National Science Foundation via the NCCR QSIT (National Center of Competence in Research, Quantum Science and Technology), the French National Research Agency ANR through projects ANR-11-LABX-0058_NIE (Labex NIE) and ANR-14-CE360007-01 (SGM-Bal), and the University of Strasbourg IdEx program.
C.R. and W.W. grew the heterostructure. T.K. and A.H. fabricated the device. R.S. designed the device. C.G. performed the measurements with support from B.A.B. C.G. and M.S.F. developed the interpretation in terms of the partial local density of states. C.G. performed the numerical simulations with support from M.S.F. K.R.F. and D.W. developed and implemented the semiclassical interpretation. D.W., K.E., and T.I. supervised the projects. All authors discussed the results.
[1] M. A. Eriksson, R. G. Beck, M. Topinka, J. A. Katine, R. M. Westervelt, K. L. Campman, and A. C. Gossard, Cryogenic scanning probe characterization of semiconductor nanostructures, Appl. Phys. Lett. 69, 671 (1996).

[2] M. A. Eriksson, R. G. Beck, M. A. Topinka, J. A. Katine, R. M. Westervelt, K. L. Campman, and A. C. Gossard, Effect of a charged scanned probe microscope tip on a subsurface electron gas, Superlattices Microstruct. 20, 435 (1996).

[3] A. C. Bleszynski, F. A. Zwanenburg, R. M. Westervelt, A. L. Roest, E. P. A. M. Bakkers, and L. P. Kouwenhoven, Scanned probe imaging of quantum dots inside InAs nanowires, Nano Lett. 7, 2559 (2007).

[4] P. Fallahi, A. C. Bleszynski, R. M. Westervelt, J. Huang, J. D. Walls, E. J. Heller, M. Hanson, and A. C. Gossard, Imaging a single-electron quantum dot, Nano Lett. 5, 223 (2005).

[5] A. E. Gildemeister, T. Ihn, M. Sigrist, K. Ensslin, D. C. Driscoll, and A. C. Gossard, Measurement of the tip-induced potential in scanning gate experiments, Phys. Rev. B 75, 195338 (2007).

[6] M. T. Woodside and P. L. McEuen, Scanned probe imaging of single-electron charge states in nanotube quantum dots, Science 296, 1098 (2002).

[7] A. Bachtold, M. S. Fuhrer, S. Plyasunov, M. Forero, E. H. Anderson, A. Zettl, and P. L. McEuen, Scanned Probe Microscopy of Electronic Transport in Carbon Nanotubes, Phys. Rev. Lett. 84, 6082 (2000).

[8] M. Bockrath, W. Liang, D. Bozovic, J. H. Hafner, C. M. Lieber, M. Tinkham, and H. Park, Resonant electron scattering by defects in single-walled carbon nanotubes, Science 291, 283 (2001).

[9] S. J. Tans and C. Dekker, Potential modulations along carbon nanotubes, Nature (London) 404, 834 (2000).

[10] M. R. Connolly, K. L. Chiu, A. Lombardo, A. Fasoli, A. C. Ferrari, D. Anderson, G. A. C. Jones, and C. G. Smith, Tilted potential induced coupling of localized states in a graphene nanoconstriction, Phys. Rev. B 83, 115441 (2011).

[11] A. G. F. Garcia, M. König, D. Goldhaber-Gordon, and K. Todd, Scanning gate microscopy of localized states in wide graphene constrictions, Phys. Rev. B 87, 085446 (2013).

[12] S. Schnez, J. Güttinger, M. Huefner, C. Stampfer, K. Ensslin, and T. Ihn, Imaging localized states in graphene nanostructures, Phys. Rev. B 82, 165445 (2010).

[13] K. E. Aidala, R. E. Parrott, T. Kramer, E. J. Heller, R. M. Westervelt, M. P. Hanson, and A. C. Gossard, Imaging magnetic focusing of coherent electron waves, Nat. Phys. 3, 464 (2007).

[14] S. Bhandari, G.-H. Lee, A. Klales, K. Watanabe, T. Taniguchi, E. Heller, P. Kim, and R. M. Westervelt, Imaging cyclotron orbits of electrons in graphene, Nano Lett. 16, 1690 (2016).
[15] M. G. Pala, B. Hackens, F. Martins, H. Sellier, V. Bayot, S. Huant, and T. Ouisse, Local density of states in mesoscopic samples from scanning gate microscopy, Phys. Rev. B 77, 125310 (2008).

[16] F. Martins, B. Hackens, M. G. Pala, T. Ouisse, H. Sellier, X. Wallart, S. Bollaert, A. Cappy, J. Chevrier, V. Bayot, and S. Huant, Imaging Electron Wave Functions Inside Open Quantum Rings, Phys. Rev. Lett. 99, 136807 (2007).

[17] F. Martins, S. Faniel, B. Rosenow, M. G. Pala, H. Sellier, S Huant, L. Desplanque, X. Wallart, V. Bayot, and B. Hackens, Scanning gate spectroscopy of transport across a quantum Hall nano-island, New J. Phys. 15, 013049 (2013).

[18] D. Cabosart, A. Felten, N. Reckinger, A. Iordanescu, S. Toussaint, S. Faniel, and B. Hackens, Recurrent quantum scars in a mesoscopic graphene ring, Nano Lett. 17, 1344 (2017).

[19] N. Pascher, C. Rössler, T. Ihn, K. Ensslin, C. Reichl, and W. Wegscheider, Imaging the Conductance of Integer and Fractional Quantum Hall Edge States, Phys. Rev. X 4, 011014 (2014).

[20] B. A. Braem, F. M. D. Pellegrino, A. Principi, M. Röösli, C. Gold, S. Hennel, J. V. Koski, M. Berl, W. Dietsche, W. Wegscheider, M. Polini, T. Ihn, and K. Ensslin, Scanning gate microscopy in a viscous electron fluid, Phys. Rev. B 98, 241304(R) (2018).

[21] M. A. Topinka, B. J. LeRoy, S. E. J. Shaw, E. J. Heller, R. M. Westervelt, K. D. Maranowski, and A. C. Gossard, Imaging coherent electron flow from a quantum point contact, Science 289, 2323 (2000).

[22] M. A. Topinka, B. J. LeRoy, R. M. Westervelt, S. E. J. Shaw, R. Fleischmann, E. J. Heller, K. D. Maranowski, and A. C. Gossard, Coherent branched flow in a two-dimensional electron gas, Nature (London) 410, 183 (2001).

[23] M. P. Jura, M. A. Topinka, L. Urban, A. Yazdani, H Shtrikman, L. N. Pfeiffer, K. W. West, and D. GoldhaberGordon, Unexpected features of branched flow through highmobility two-dimensional electron gases, Nat. Phys. 3, 841 (2007).

[24] K. Kolasiński and B. Szafran, Simulations of imaging of the local density of states by a charged probe technique for resonant cavities, Phys. Rev. B 88, 165306 (2013).

[25] O. Ly, R. A. Jalabert, S. Tomsovic, and D. Weinmann, Partial local density of states from scanning gate microscopy, Phys. Rev. B 96, 125439 (2017).

[26] R. Steinacher, C. Pöltl, T. Krähenmann, A. Hofmann, C. Reichl, W. Zwerger, W. Wegscheider, R. A. Jalabert, K. Ensslin, D. Weinmann, and T. Ihn, Scanning gate experiments: From strongly to weakly invasive probes, Phys. Rev. B 98, 075426 (2018). 
[27] N. Aoki, R. Brunner, A. M. Burke, R. Akis, R. Meisels, D. K. Ferry, and Y. Ochiai, Direct Imaging of Electron States in Open Quantum Dots, Phys. Rev. Lett. 108, 136804 (2012).

[28] D. K. Ferry, A. M. Burke, R. Akis, R. Brunner, T. E. Day, R. Meisels, F. Kuchar, J. P. Bird, and B. R. Bennett, Open quantum dots-Probing the quantum to classical transition, Semicond. Sci. Technol. 26, 043001 (2011).

[29] R. Steinacher, A. A. Kozikov, C. Rössler, C. Reichl, W. Wegscheider, K. Ensslin, and T. Ihn, Scanning gate imaging in confined geometries, Phys. Rev. B 93, 085303 (2016).

[30] C. Yan, S. Kumar, M. Pepper, P. See, I. Farrer, D. Ritchie, J. Griffiths, and G. Jones, Interference Effects in a Tunable Quantum Point Contact Integrated with an Electronic Cavity, Phys. Rev. Appl. 8, 024009 (2017).

[31] C. Yan, S. Kumar, M. Pepper, P. See, I. Farrer, D. Ritchie, J. Griffiths, and G. Jones, Incipient singlet-triplet states in a hybrid mesoscopic system, Phys. Rev. B 97, 241302(R) (2018).

[32] C. Yan, S. Kumar, P. See, I. Farrer, D. Ritchie, J. P. Griffiths, G. A. C. Jones, and M. Pepper, Magnetoresistance in an electronic cavity coupled to one-dimensional systems, Appl. Phys. Lett. 113, 112101 (2018).

[33] C. Rössler, D. Oehri, O. Zilberberg, G. Blatter, M. Karalic, J. Pijnenburg, A. Hofmann, T. Ihn, K. Ensslin, C. Reichl, and W. Wegscheider, Transport Spectroscopy of a Spin-Coherent DotCavity System, Phys. Rev. Lett. 115, 166603 (2015).

[34] See Supplemental Material at http://link.aps.org/supplemental/ 10.1103/PhysRevResearch.3.L032005 for (Sec. SI) further experimental details, (Sec. SII) the cavity mode spacing, (Sec. SIII) the estimation of the number of populated cavity modes and their energy spacing, (Sec. SIV) the Fourier transformation of the conductance modulations in the tip and cavity voltage, (Sec. SV) further measurements of the conductance modulations as a function of the cavity and tip voltage as well as the tip position, (Sec. SVI) the implementation of the smooth disorder potential, (Sec. SVII) the numerical measurement for a Gaussian tip potential, (Sec. SVIII) the statistics of the average difference in the partial local density of states, and (Sec. SIX) a brief description of a possible experiment to extract the local density of states from SGM measurements.

[35] M. S. Ferguson, D. Oehri, C. Rössler, T. Ihn, K. Ensslin, G. Blatter, and O. Zilberberg, Long-range spin-coherence in a strongly-coupled all electronic dot-cavity system, Phys. Rev. B 96, 235431 (2017).

[36] E. J. Heller and S. Shaw, Branching and Fringing in Microstructure Electron Flow, Int. J. Mod. Phys. B 17, 3977 (2003).
[37] C. Pöltl, A. Kozikov, K. Ensslin, T. Ihn, R. A. Jalabert, C. Reichl, W. Wegscheider, and D. Weinmann, Classical origin of conductance oscillations in an integrable cavity, Phys. Rev. B 94, 195304 (2016).

[38] K. R. Fratus, R. A. Jalabert, and D. Weinmann, Energy stability of branching in the scanning gate response of two-dimensional electron gases with smooth disorder, Phys. Rev. B 100, 155435 (2019).

[39] L. Bours, S. Guiducci, A. Mreńca-Kolasińska, B. Szafran, J. C. Maan, and S. Heun, Manipulating quantum Hall edge channels in graphene through scanning gate microscopy, Phys. Rev. B 96, 195423 (2017).

[40] A. Mreńca-Kolasińska and B. Szafran, Imaging backscattering in graphene quantum point contacts, Phys. Rev. B 96, 165310 (2017).

[41] B. Brun, N. Moreau, S. Somanchi, V.-H. Nguyen, K. Watanabe, T. Taniguchi, J.-C. Charlier, C. Stampfer, and B. Hackens, Imaging Dirac fermions flow through a circular Veselago lens, Phys. Rev. B 100, 041401(R) (2019)

[42] K. R. Fratus, C. Le Calonnec, R. Jalabert, G. Weick, and D. Weinmann, Signatures of folded branches in the scanning gate microscopy of ballistic electronic cavities, SciPost Phys. 10, 069 (2021).

[43] C. W. Groth, M. Wimmer, A. R. Akhmerov, and X. Waintal, Kwant: A software package for quantum transport, New J. Phys. 16, 063065 (2014)

[44] J. H. Davies, I. A. Larkin, and E. V. Sukhorukov, Modeling the patterned two-dimensional electron gas: Electrostatics, J. Appl. Phys. 77, 4504 (1995).

[45] R. Steinacher, A. A. Kozikov, C. Rössler, C. Reichl, W. Wegscheider, T. Ihn, and K. Ensslin, Scanning-gate-induced effects and spatial mapping of a cavity, New J. Phys. 17, 043043 (2015).

[46] The partial local density of states originating from the source contact is the contribution of the scattering states impinging from that contact to the local density of states (see, e.g. $[25,48,49])$.

[47] See Supplemental Material at http://link.aps.org/supplemental/ 10.1103/PhysRevResearch.3.L032005 for movies.

[48] T. Gramespacher and M. Büttiker, Local densities, distribution functions, and wave-function correlations for spatially resolved shot noise at nanocontacts, Phys. Rev. B 60, 2375 (1999).

[49] M. Büttiker and T. Christen, Basic elements of electrical conduction, in Quantum Transport in Semiconductor Submicron Structures, NATO ASI Series, edited by B. Kramer (Springer Netherlands, Dordrecht, 1996), pp. 263-291. 